\title{
In-situ fabrication of polymer microsieves for $\mu$ TAS by slanted angle holography
}

\author{
An M. Prenen · Anja Knopf • Cees W. M. Bastiaansen • \\ Dirk J. Broer
}

Received: 23 November 2010/ Accepted: 6 December 2010/Published online: 29 December 2010

(C) The Author(s) 2010. This article is published with open access at Springerlink.com

\begin{abstract}
We present a simple, versatile method for the in-situ fabrication of membranes inside a microfluidic channel during a chip manufacturing process using only two extra slanted angle holographic exposure steps. This method combines the strengths of both inclined UV exposure and holographic lithography to produce micrometer-sized three-dimensional sieving structures. Using a common chip material, the photoresist material SU-8, together with this method, a leak-free membranechannel connection is obtained. The resulting membranes are monodisperse, with a very well-defined pore geometry (i.e., microsieves with a pore diameter between $500 \mathrm{~nm}$ and $10 \mu \mathrm{m}$ ) that is easily controllable with the holographic set-up. The selectivity of in-situ fabricated microsieves with a pore diameter of $2 \mu \mathrm{m}$ will be demonstrated using polystyrene beads of 1 and $3 \mu \mathrm{m}$.
\end{abstract}

Keywords Holography · Microsieves - Microfluidics · In-situ fabrication · Inclined lithography $\cdot$ SU-8

A. M. Prenen $(\bowtie) \cdot$ C. W. M. Bastiaansen - D. J. Broer Eindhoven University of Technology, Post Box 513, 5600 MB Eindhoven, The Netherlands

e-mail: anprenen@gmail.com

\section{A. Knopf}

Johannes Gutenberg-Universität Main, 55099 Mainz, Germany

C. W. M. Bastiaansen

Queen Mary University of London, Mile End Road,

London E1 4NS, UK

\section{Introduction}

Microfluidic devices are extensively investigated for the control and manipulation of small fluid volumes (Gravesen et al. 1993). Usually, a microfluidic device is defined as a device having at least one channel with a diameter of less than $1 \mathrm{~mm}$. In biosensors, these devices are often used to process small quantities of fluid and/or to detect the presence of chemical or biological substances in a small fluid volume (Erickson and Li 2004). Typical fluids in microfluidics are: whole blood, blood plasma, saliva, urine, protein or antibody solutions and various buffers. These are complex mixtures that need various treatments such as filtering/purification, mixing and chemical treatment. Often, one or more membranes are therefore incorporated in a microfluidic device (De Jong et al. 2006). Its function can be diverse including separation of particles from liquids, induction of turbulence for mixing and/or providing of a large internal surface area for adsorption.

Frequently, commercially available membranes are implemented in microfluidics (Thorslund et al. 2006). Typically, these membranes are non-woven fabrics and exhibit a highly polydisperse pore structure and high porosity. Consequently, the application of these membranes results in a large pressure drop and restricts the throughput of the fluid in the device (Baker 2004). Monodisperse microsieves (usually in silicon) have a low pressure drop (Kuiper et al. 1998; Heyderman et al. 2003) but the actual implementation in a microfluidic device is complicated and laborious. Moreover, the sealing of devices with microsieves often poses a serious problem (De Jong et al. 2006).

Both inclined lithographic exposure and holographic lithography are well-established techniques for the fabrication of microstructures. Holographic lithography excels 
in producing very small features (Kondo et al. 2006; Romanato et al. 2006) and even membranes (Prenen et al. 2009), while inclined exposures form an easy method to create somewhat larger $(>100 \mu \mathrm{m})$ structures (Sato et al. 2003; Han et al. 2004). Here, we present a new method for the in-situ fabrication polymer microsieves inside a microfluidic channel which combines these two techniques, and therefore, takes advantages of the strengths of both. This enables us to produce three-dimensional microsieving structures with well-controlled pore diameter in the micrometer range. Inclined holographic exposure through a mask will result in functional microsieves which are chemically (and hence leak-free) connected to the microchannel at discrete positions where they will be tested on their performance. The production of the microsieves is based on the use of lithographic and holographic processes which are common processes in the production of microfluidic devices.

The material used in this study is SU-8 (Shaw et al. 1997), a photoresist that is frequently used as a chip material for micro-total analysis systems ( $\mu$ TAS) (Zhang et al. 2001; Mogensen et al. 2003; Chuang et al. 2003). It will be shown that the addition of a few clever exposure steps to the photolithographic process is needed to obtain monodisperse microsieves incorporated in a microfluidic device. The great advantage of this approach is that sealing at the channel walls is not an issue, since the membrane is made of the same material and during the same processing step as the chip itself.

\section{Experimental}

SU-8, a commercially available negative photoresist (MicroChem Corporation) is used as chip material in this study. It has a strong non-linear optical response which results in a digital recording of the analogue interference pattern (Shaw et al. 1997). Moreover, the photoresist mixture is in the glassy state at room temperature, minimizing mobility during irradiation, and therefore, enabling the utilization of several consecutive exposure steps.

Glass slides are cleaned in ethanol and dried with nitrogen gas. A $50 \mathrm{~nm}$ thin layer of Omnicoat (Microresist Corp.) is spin coated $(4,000 \mathrm{rpm}, 20 \mathrm{~s})$ and cured (2 min at $200^{\circ} \mathrm{C}$ ) to ensure a good adhesion of the SU-8 to the glass substrate. A layer of SU-8 is spin coated (SU-8-2010, for layers up to $10 \mu \mathrm{m}$ ) or doctor bladed (SU-8-2100, for layers between 10 and $100 \mu \mathrm{m}$ thickness) on a cleaned glass substrate. Thereafter, a 2-step pre-bake is performed to evaporate the solvents and to ensure that the photoresist is in the glassy state. The heating times for the two layer thicknesses used in this study, 6 and $50 \mu \mathrm{m}$, are displayed in Table 1. Note that for the $6 \mu \mathrm{m}$ film, these values are
Table 1 Heating times for pre and post-exposure bake

\begin{tabular}{lcclcc}
\hline Film & \multicolumn{2}{l}{ Prebake } & & Postbake & Development \\
\cline { 2 - 3 } $\begin{array}{l}\text { Thickness } \\
(\mu \mathrm{m})\end{array}$ & $\begin{array}{l}65^{\circ} \mathrm{C} \\
(\min )\end{array}$ & $\begin{array}{l}95^{\circ} \mathrm{C} \\
(\min )\end{array}$ & $\begin{array}{l}65^{\circ} \mathrm{C} \\
(\min )\end{array}$ & $\begin{array}{l}95^{\circ} \mathrm{C} \\
(\min )\end{array}$ & Time $(\min )$ \\
\hline 6 & 1 & 2 & 1 & 2 & $<1$ \\
50 & 30 & 60 & 1 & 15 & 5 \\
\hline
\end{tabular}

equal to these in the SU-8 datasheet, while for the $50 \mu \mathrm{m}$ film significantly different times were obtained in an optimization procedure.

The UV exposures are carried out using the $351 \mathrm{~nm}$ line of a CW $\mathrm{Ar}^{+}$ion laser (Beamlok 2080-25S, SpectraPhysics). The laser beam is guided through a spatial filter formed by 2 lenses and a pinhole to filter out inhomogeneities of the beam intensity (Fig. 1a). A $\lambda / 2$ plate rotates the beams' polarization to $45^{\circ}$, after which it is split into one horizontally polarized and one vertically polarized beam by a polarizing beam splitter (PBS). Another $\lambda / 2$ plate in the transmitted beam rotates the polarization of that beam to vertical. The sinusoidal interference pattern generated by the resulting two parallel polarized crossing laser beams is used for the holographic irradiation. The periodicity $\Lambda$ of this pattern depends on the laser wavelength $\lambda$, the angle between the laser beam $\theta$ and the average refractive index of the recording material $n$, according to Bragg's law (Bragg 1913).

$\Lambda=\frac{\lambda}{2 n \sin \theta / 2}$

Usually, a photoresist is illuminated with an interference pattern where the grating vector is perpendicular to the

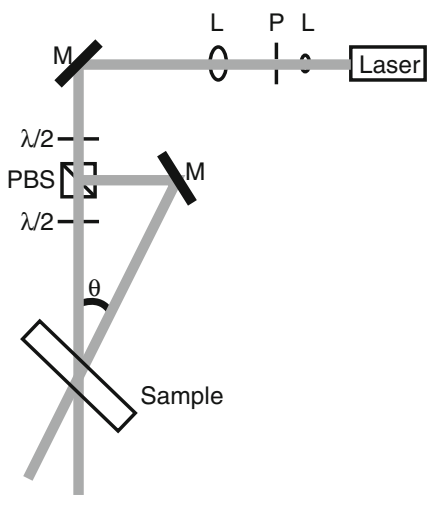

(a)

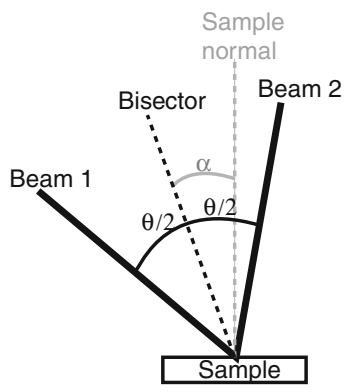

(b)
Fig. 1 a Schematic overview of the slanted angle holographic set-up. A laser beam is filtered by a spatial filter consisting of 2 lenses $(\mathrm{L})$ and a pinhole $(\mathrm{P})$, directed to a $\lambda / 2$ plate by a mirror, passes through a polarizing beam splitter (PBS) where it is split into 2 perpendicularly polarized beams. The transmitted beam passes through a second $\lambda / 2$ plate, after which it is directed to the sample, crossing the other beam at an angle $\theta$. $\mathbf{b}$ detailed schematic view of the geometry of a slanted angle holographic exposure with a tilt angle $\alpha$ 


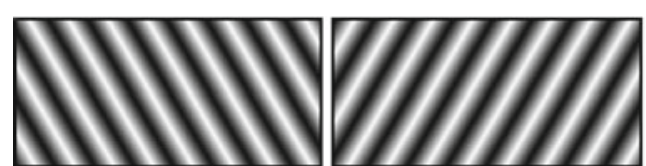

(a) (b)

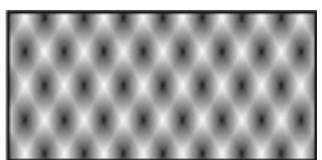

(c)

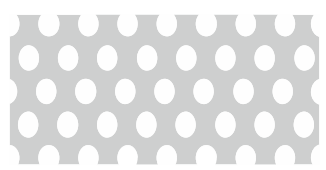

(d)

Fig. 2 Schematic representation of the latent image formation during slanted angle holography. a exposure over tilt angle $\alpha, \mathbf{b}$ exposure over tilt angle $-\alpha$, $\mathbf{c}$ resulting latent image, $\mathbf{d}$ resulting structure after crosslinking and development

sample normal. Here, the photoresist is exposed with the sample tilted over an angle a with respect to the bisector of the angle between the two interfering laser beams (Fig. 1b), as used in, e.g., the fabrication of slanted gratings (Van Heesch et al. 2005).

The latent image produced by such an exposure is depicted in Fig. 2. Areas of high and low intensities lie in lamellae-like structures that are tilted over the same angle $\alpha$ (Fig. 2a). Likewise, a second exposure step, this time with a tilt angle $-\alpha$ results in lamellae tilted over $-\alpha$ (Fig. $2 b$ ). The resulting latent image of the final structure then shows a honeycomb-like shape, as can be found in Fig. 2c. During illumination a Lewis acid is formed in the high intensity areas, which acts as a catalyst for the cationic polymerization. Crosslinking is induced during a second heating sequence (post-exposure bake (PEB), for temperatures and times see: Table 1) which is followed by slowly cooling down the sample to minimize buildup of internal stresses due to temperature differences between the glass substrate and the SU-8. The final structure is obtained when unreacted monomer is rinsed out of the non-exposed parts by consecutively immersing the sample in SU-8 developer (mr-dev 600, MicroChem Corp.) and in propanol-2 after which it is dried with nitrogen gas (Fig. 2d).

For scanning electron microscopy (SEM) analysis (XL 30 ESEM-FEG, Philips), a 15 nm layer of gold (K575 XD Turbo sputter coater, Emitech, Ltd.) is sputter coated on the sample after fabrication.

The filtration capacity of the membranes fabricated inside the microchannels are characterized using a dispersion of dark red polystyrene microspheres (Fluka). The filtration experiments are monitored with an optical microscope (Leica DM6000M, equipped with a DFC420 camera).

\section{Results and discussion}

\subsection{Fabrication}

In a first set of experiments, the possibility to produce inplane membranes with a variety of pore sizes and shapes is explored. Therefore, samples with a $6 \mu \mathrm{m}$ film of SU-8 on a glass substrate coated with Omnicoat are prepared and consecutively irradiated with a tilt angle of $\alpha$ and $-\alpha$ with respect to the interference pattern of the intersecting laser beams, followed by PEB and development.

By changing the periodicity of the interference pattern, the pore size of the resulting membrane is adaptable to the desired diameter. In Fig. 3 this is illustrated by 3 crosssectional SEM images of three in-plane membranes, exposed to interference patterns with periodicities of, respectively, 1,2 , and $7 \mu \mathrm{m}$, corresponding to $\theta$ between the beams of, respectively, $20^{\circ}, 10^{\circ}$, and $4^{\circ}$, all membranes are fabricated with a tilt angle $\alpha$ of $30^{\circ}$. It is observed that the recording of small periodicities (e.g., $1 \mu \mathrm{m}$ ) results in the formation of membranes with smaller pore diameters, in multiple layers on top of each other.

Upon exposure to a different tilt angle $\alpha$, the pore shape and array are influenced. Smaller tilt angles are expected to result in slit-shaped pores, while large tilt angles should give more round pores. Slit-shaped pores have many advantages over round pores (Kuiper et al. 2002). For example, the open area of a membrane with slit-shaped

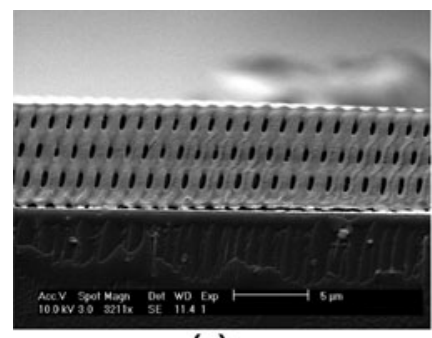

(a)

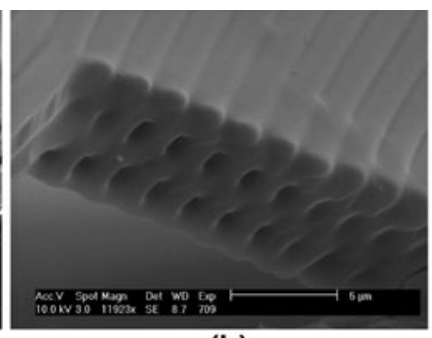

(b)

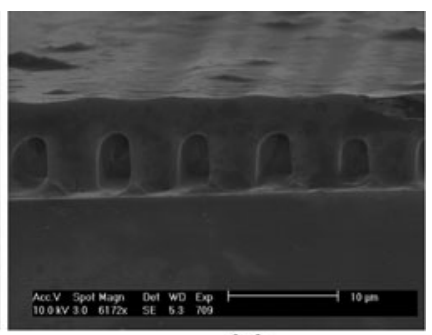

(c)

Fig. 3 Cross-section SEM images of in-plane membranes fabricated with different periodicities of the interference pattern: a $1 \mu \mathrm{m}$, b $2 \mu \mathrm{m}$, and $\mathbf{c} 7 \mu \mathrm{m}$ 


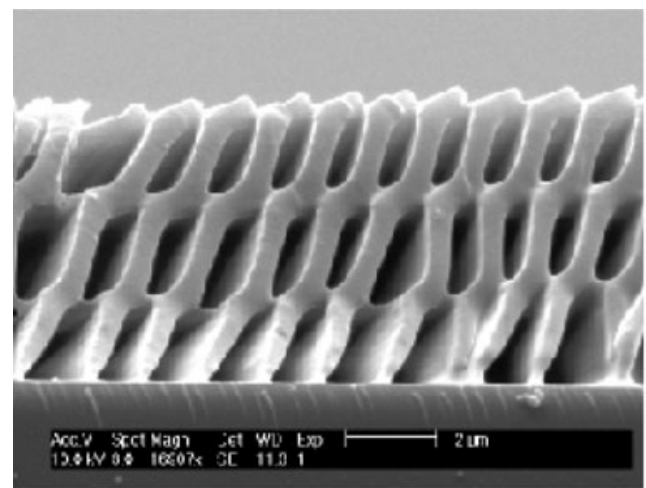

(a)

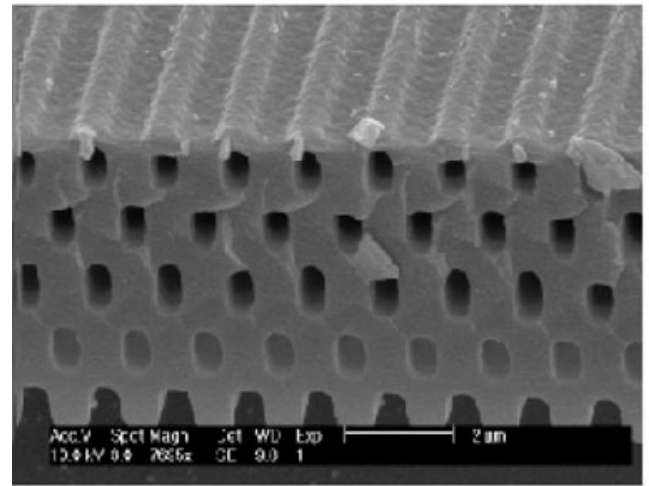

(c)

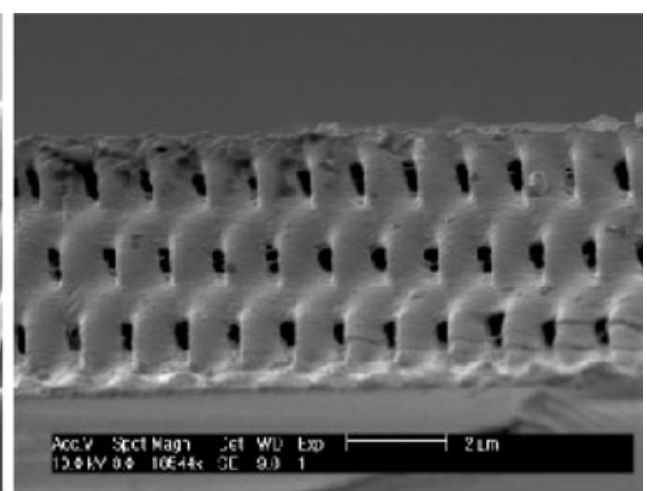

(b)

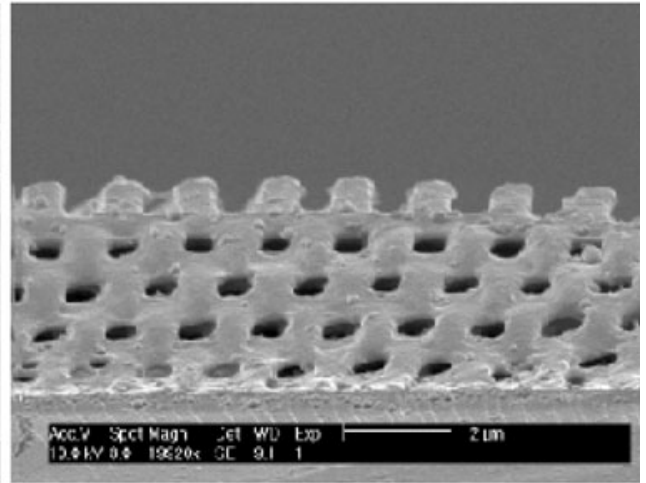

(d)

Fig. 4 Cross-section SEM images of membranes fabricated using a periodicity of $1 \mu \mathrm{m}$ and tilt angles of $\mathbf{a} 10^{\circ}$, b $20^{\circ}$, c $30^{\circ}$, and d $40^{\circ}$. It can be seen from the images that the angle $\alpha$ influences the pore shape from slit-shaped (small $\alpha$ ) to round or even square shaped (large $\alpha$ )

pores is larger than that of a membrane with round pores, having the same selectivity, i.e., the same smallest pore diameter. Therefore, the pressure drop of such a slit-shaped membrane is lower than that of a membrane with round pores. Moreover, slit-shaped pores are less susceptible to fouling, due to the lower surface to volume ratio of the pores.

In Fig. 4, the effect of changing the tilt angle is clearly illustrated by cross-section SEM images of membranes where $\alpha=10^{\circ}, 20^{\circ}, 30^{\circ}, 40^{\circ}$, for a periodicity of $1 \mu \mathrm{m}$. From the SEM images, it can be found that the minimum diameter of the pores of all four fabricated membranes is approximately equal. This indicates that all four membranes have the same selectivity.

In the second set of experiments, the in-plane membranes described in the previous paragraph are fabricated in situ in a microfluidic channel. To achieve this, a glass substrate is coated with a thin layer of SU-8, typically $5 \mu \mathrm{m}$, which is flood exposed $\left(200 \mathrm{~mJ} / \mathrm{cm}^{2}\right)$ and postbaked. This acts as an adhesion layer to which the microfluidic channel and the membrane will be chemically bonded. A $50 \mu \mathrm{m}$ thick film is doctor bladed and prebaked. After cooling down, it is first exposed to UV-light $(351 \mathrm{~nm})$ through a line mask containing the negative image of the microfluidic channels with a width of $75 \mu \mathrm{m}$. In a consecutive exposure step, the slanted-angle holographic exposure is performed through a line mask with a smaller periodicity $(10 \mu \mathrm{m})$ is performed. The sample is brought into optical contact with the mask using cyclohexane as a contact fluid, to avoid reflections at the SU-8/ mask interface. The dimensions of the transparent lines are typically in the micrometer range, in this case $5 \mu \mathrm{m}$, and they correspond to the final thickness of the membranes in the microfluidic channel. The holographic exposure step is performed with periodicity of $2 \mu \mathrm{m}$ and a tilt angle of $40^{\circ}$. After exposure, the sample is crosslinked (PEB), followed by the development step.

An example of a sample containing equally spaced microfluidic channels containing a multitude of in-plane membranes is shown in the SEM images of Fig. 5. The pore spacing used to make these membranes is $2 \mu \mathrm{m}$ and the angle $\alpha$ is $40^{\circ}$, with a resulting minimum pore diameter of $720 \mathrm{~nm}$. In the SEM image of Fig. 5b the sharp contours of the well-defined membranes are displayed. From this image it can also be observed that both the microfluidic channel walls and the membrane itself are physically attached to each other-which is expected, as they are fabricated in the very same process. Also the connection of 


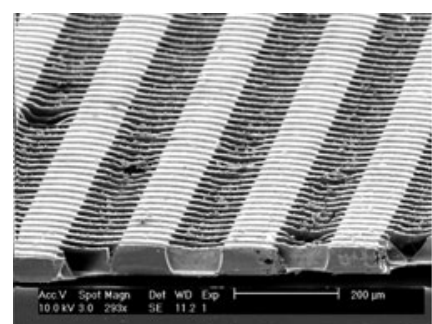

(a)

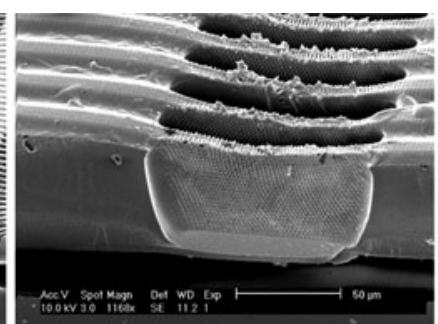

(b)

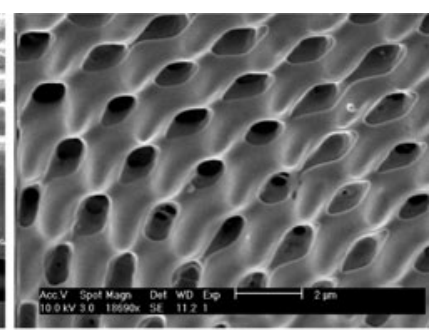

(c)

Fig. 5 SEM images of in-situ fabricated microfluidic filters. a overview, b zoom in of four membranes inside channel, c zoom in of membrane: pores are open and have narrow pore size distribution with a minimum pore diameter of $720 \mathrm{~nm}$

the membrane to the SU-8 adhesion layer on the bottom looks impeccable. This gives a good indication that the structures are leak-proof. From Fig. 5c, it is clearly visible that the membrane pores are open and the pore size distribution is very small.

Since an sinodal intensity pattern is used for the illumination of the microsieve structures, for large film thicknesses $(>100 \mu \mathrm{m})$ the UV-absorption of the SU-8 would lead to the formation of larger pores on the nonillumination side of the sample. Then, a gradient in minimum pore diameter would be observed and hence the selectivity would deteriorate.

\subsection{Characterization}

Three samples with $50 \mu \mathrm{m}$ wide channels $(50 \mu \mathrm{m}$ high, square cross-section, open top) containing $15 \mu \mathrm{m}$ wide membranes with a minimum pore diameter of $0.5,2.5$, or $4 \mu \mathrm{m}$, are capillary filled with a dispersion of 1 or $3 \mu \mathrm{m}$ (or both sizes mixed) polystyrene microbeads (Fluka). The experiments were performed with open channels, due to the fact that sealing them with a PDMS slab and a rigid support gave a too high stack to visualize in the microscope. However, this sealing method was observed to leave the membrane intact after removal of the PDMS.

In Fig. 6a, a microscope image of the membrane with a minimum pore diameter of $2.5 \mu \mathrm{m}$ which is filled with a mixture of 1 and $3 \mu \mathrm{m}$ beads in water is shown. The fluid flows from right to left, and it is clear that the $3 \mu \mathrm{m}$ beads are retained by the membrane, while the $1 \mu \mathrm{m}$ beads are allowed to pass through the membrane.

Also, the $0.5 \mu \mathrm{m}$ membrane and the $4 \mu \mathrm{m}$ membrane are tested on their filtration characteristics. In Fig. $6 \mathrm{~b}$ it can be seen that the $1 \mu \mathrm{m}$ beads are retained by the $0.5 \mu \mathrm{m}$ pores of the membrane. Figure $6 \mathrm{c}$ shows that the $3 \mu \mathrm{m}$ beads can pass through the $4 \mu \mathrm{m}$ membrane pores, they are present on both sides of the membrane while the flow comes from the right, even though a cluster of beads blocks the membrane partially.

From these experiments it can be concluded that the physical presence of these in-plane membranes, inside a

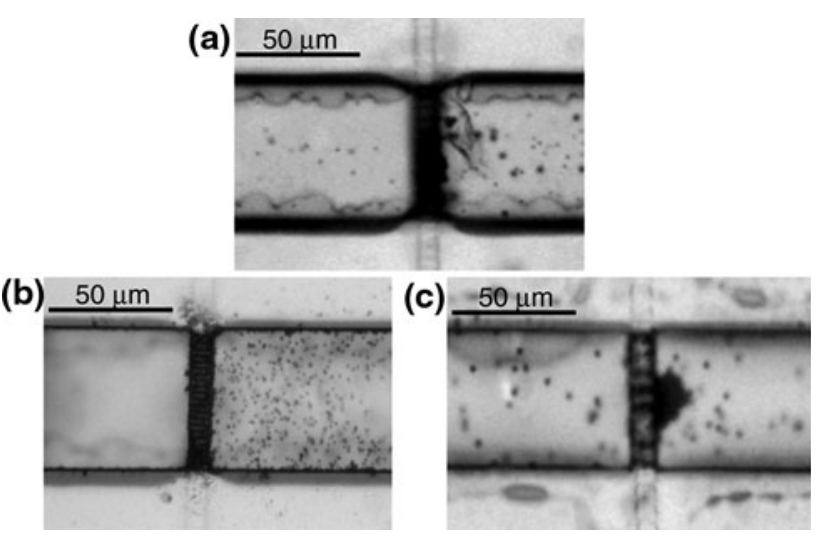

Fig. 6 a Microscope image of capillary filling of a $50 \mu \mathrm{m}$ wide channel containing a $15 \mu \mathrm{m}$ wide membrane with a minimum pore diameter of $2.5 \mu \mathrm{m}$, with a dispersion of 1 and $3 \mu \mathrm{m}$ polystyrene microbeads. The $3 \mu \mathrm{m}$ beads are retained and the $1 \mu \mathrm{m}$ beads pass through. b Optical microscope images of filtration experiments, microchannels are capillary filled from the right. $1 \mu \mathrm{m}$ microspheres are retained on the right side of a membrane with minimum pore diameter $0.5 \mu \mathrm{m}$. c $3 \mu \mathrm{m}$ microspheres present on both sides of the membrane with a minimumpore diameter of $4 \mu \mathrm{m}$

microfluidic channel provides the channel with structures having a large specific surface, e.g., for adsorption of biomolecules. And additionally, they can also fulfill the filtration function they were originally designed for, in a very efficient, selective way.

The flow resistance of the membranes is determined by measuring the face velocity of water before and after it has passed a membrane during capillary filling of a channel. For this experiment, a $50 \mu \mathrm{m}$ thick sample is used, in which $50 \mu \mathrm{m}$ wide channels are fabricated using mask lithography, containing a $25 \mu \mathrm{m}$ wide membrane with a pore size of approximately $2 \mu \mathrm{m}$. Fifteen channels are capillary filled with water, and the face velocity is determined using optical microscopy. The average initial face velocity for the water is measured to be $95 \pm 10 \mathrm{~mm} / \mathrm{s}$. The deviation on this is rather large due to the relatively low frame rate (100 frames per second) of the camera on the microscope. The face velocity of the water after passing the membrane is determined as $16 \pm 4 \mathrm{~mm} / \mathrm{s}$. Although 
this is a reduction of a factor of 6 in the flux, it needs to be taken into account that the membrane used for this test had an aspect ratio (membrane thickness/pore diameter) of 12.5 , while in an ideal case the microsieve would have an aspect ratio of 1 (Kuiper et al. 2002). Decreasing the membrane thickness would reduce the flow resistance of the microsieve significantly. Since the thickness of the membrane is dependent on the dimensions of the mask used, a microsieve thickness of $2 \mu \mathrm{m}$ would be the lower limit of this fabrication technique.

\section{Conclusions}

The results described above show that the combination of SU-8, a common chip material, and slanted angle holography creates an exceptional opportunity to produce monodisperse microfilters for micro total analysis systems in situ, using a set of cleverly chosen exposure steps. Membranes with a narrow pore size distribution are easily fabricated. Pore dimensions are shown to be controllable 0.5 and $3 \mu \mathrm{m}$ by changing the angles $\alpha$ and $\theta$. Furthermore, the monodisperse microsieves are manufactured in situ inside the microchannels using slanted angle holography during the chip fabrication process itself. This approach results in a leak-proof connection between the channel walls, the channel bottom and the membranes. The filtration capabilities of the in-situ fabricated membranes are tested using a combination of a colored fluid and microspheres. The microspheres, having a diameter larger than the minimum pore diameter are shown to be retained well by the membranes.

The above described approach to the in-situ fabrication of membranes in microchannels is believed to provide an exceptional design freedom for the leak-free implementation of filtration functionalities into $\mu$ TAS.

Open Access This article is distributed under the terms of the Creative Commons Attribution Noncommercial License which permits any noncommercial use, distribution, and reproduction in any medium, provided the original author(s) and source are credited.

\section{References}

Baker RW (2004) Membrane technology and applications, 2nd edn. Wiley, UK
Bragg WL (1913) The diffraction of short electromagnetic waves by a crystal. Proc Camb Philos Soc 17:43-57

Chuang Y-J, Tseng F-G, Cheng J-H, Lin W-K (2003) A novel fabrication method of embedded micro channels by using SU-8 thick film photoresists. Sens Actuators A 103:64-69

De Jong J, Lammertink RGH, Wessling M (2006) Membranes and microfluidics: a review. Lab Chip 6:1125-1139

Erickson D, Li D (2004) Integrated microfluidic devices. Anal Chim Acta 507:11-26

Gravesen G, Branebjerg J, Jensen OS (1993) Microfluidic-a review. J Micromech Microeng 3:168-182

Han M, Lee W, Lee S-K, Lee S (2004) 3D microfabrication with inclined/rotated UV lithography. Sens Actuator A 111:14-20

Heyderman LJ, Ketterer B, Bächle S et al (2003) High volume fabrication of customised nanopore membrane chips. Microelectron Eng 67-68:208-213

Kondo T, Juodzakis S, Mizeikis V, Misawa H, Matsuo S (2006) Holographic lithography of periodic two- and three-dimensional microstructures in photoresist SU-8. Opt Express 14(17): $7943-7953$

Kuiper S, van Rijn CJM, Nijdam W, Elwenspoek MC (1998) Developments and applications of very high flux micro filtration membranes. J Membr Sci 150:1-8

Kuiper S, Brink R, Nijdam W, Krijnen GJM, Elwenspoek MC (2002) Ceramic microsieves: influence of perforation shape and distribution on flow resistance and membrane strength. J Membr Sci 196(2):149-157

Mogensen KB, Eli-Ali J, Wolff A, Kutter JP (2003) Integration of polymer waveguides for optical detection in microfabricated chemical analysis systems. Appl Opt 42(19):4072-4079

Prenen AM, van der Werf JCA, Bastiaansen CW M, Broer DJ (2009) Monodisperse polymeric micro and nanosieves produced with interference lithography. Adv Mater 21(17):1751-1755

Romanato F, Businaro L, Tormen M, Perennes F, Matteucci M, Marmiroli B, Balslev S, Di Fabrizio E (2006) Fabrication of 3D micro and nanostructures for MEMS and MOEMS: an approach based on combined lithographies. J Phys Conf Ser 34:904-911

Sato H, Kakinuma T, Go JS, Shoji S (2003) A novel fabrication of inchannel 3-D micromesh structure using maskless multi-angle exposure and its microfilter application. 16th International conference on micro electro mechanical systems: MEMS2003, Kyoto, pp 223-226

Shaw M, Gelorme JD, LaBianca NC et al (1997) Negative photoresists for optical lithography. IBM J Res Dev 1997(41): 81-94

Thorslund S, Klett O, Nikolajeff F, Markides K, Bergquist J (2006) A hybrid poly(dimethylsiloxane) microsystem for on-chip whole blood filtration optimized for steroid screening. Biomed Microdevices 18:419

Van Heesch CM, Jagt HJB, Sanchez C, Cornelissen HJM, Broer DJ, Bastiaansen CWM (2005) Polarized light outcoupling from lightguides for LCDs. Chem Rec 5(2):59-69

Zhang J, Tan KL, Hong GD, Yang LJ, Gong HQ (2001) Polymerization optimization of SU-8 photoresist and its applications in microfluidic systems and MEMS. J Micromech Microeng 11:20-26 